\title{
An elevated plasma level of visfatin increases the risk of myocardial infarction
}

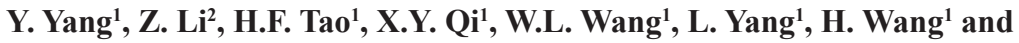
P. $\mathrm{Xu}^{1}$

${ }^{1}$ Department of Cardiovascular Medicine,

Shenzhou Hospital of Shenyang Medical University, Shenyang, China

${ }^{2}$ Department of Cardiovascular Medicine,

The First Affiliated Hospital of China Medical University, Shenyang, China

Corresponding author: P. Xu

E-mail: szyy_xp@126.com

Genet. Mol. Res. 13 (4): 8586-8595 (2014)

Received July 10, 2013

Accepted November 29, 2013

Published January 24, 2014

DOI http://dx.doi.org/10.4238/2014.January.24.18

\begin{abstract}
Visfatin, an adipocytokine involved in metabolic and immune disorders, plays an important role in the etiology of cardiovascular disease. Recent evidence has shown that an elevated plasma level of visfatin may increase the risk of myocardial infarction (MI), but individual published studies have shown inconclusive results. This study aimed to obtain a more precise estimate of the association between the plasma visfatin level and MI risk through a detailed meta-analysis of studies published in peer-reviewed journals. A literature search of articles published before May 1, 2013 was performed on the PubMed, Embase, Web of Science, and China BioMedicine databases. Crude standardized mean differences (SMDs) with $95 \%$ confidence intervals (CI) were calculated. Eleven case-control studies comprising 362 MI patients and 322 healthy controls were included. The meta-analysis revealed that an elevated plasma level of visfatin was associated with an increased risk of MI $(\mathrm{SMD}=3.82,95 \% \mathrm{CI}=2.67-4.98, \mathrm{P}<0.001)$. Further stratification based on the source of the controls showed that an elevated plasma
\end{abstract}


level of visfatin was significantly associated with increased risk of MI in both hospital-based and population-based studies (SMD = $4.12,95 \% \mathrm{CI}=2.23-6.01, \mathrm{P}<0.001$ and $\mathrm{SMD}=3.65,95 \% \mathrm{CI}=2.67-$ $4.98, \mathrm{P}<0.001$, respectively). No publication bias was evident in this meta-analysis. In conclusion, the current meta-analysis indicates that an elevated plasma level of visfatin increases the risk of MI. Therefore, plasma visfatin may be a promising biomarker for the diagnosis of MI.

Key words: Visfatin; Cardiovascular disease; Myocardial infarction; Meta-analysis

\section{INTRODUCTION}

Myocardial infarction (MI) is a major cause of mortality and morbidity worldwide, accounting for up to $40 \%$ of all deaths (Yeh et al., 2010; Insam et al., 2013). Atherosclerotic inflammation is the critical factor in both the formation of coronary plaque and the progression of the plaque to an unstable state that leads to MI (Stone et al., 2011). Obesity is known to increase the oxygen consumption required for a given workload and thereby predispose affected individuals to developing cardiovascular disease (CAD) (Akerman et al., 2004; Tilg and Moschen, 2006). Obesity is of paramount importance as a risk factor for MI (Freedman et al., 2001; Stephen and Janssen, 2009). Excessive body fat can secrete a variety of bioactive substances, termed adipokines; these include growth factors, cytokines, and complement (Guerre-Millo, 2004). Emerging evidence indicates that adipose tissue promotes systemic vascular inflammation in an endocrine manner (Berg and Scherer, 2005; Iacobellis and Bianco, 2011). Adipokines have been demonstrated to regulate various stages of atherosclerosis from endothelial dysfunction to plaque destabilization and rupture (Zhang et al., 2010).

Visfatin is a newly identified proinflammatory adipokine that has been linked to CAD, including MI (Kadoglou et al., 2011). Visfatin is a 52- to 55-kDa protein that was originally cloned as a growth factor that enhanced the effects of stem cell factor and IL-7 on colony formation by early-stage B cells (Yan et al., 2010). Visfatin is found mainly in visceral adipose tissue and mimics insulin in its suppression of the plasma glucose level (Wang et al., 2009). Recent studies have indicated that the plasma visfatin level correlates with the serum levels of CRP, IL-6, and TNF- $\alpha$, indicating that circulating visfatin may reflect an individual's inflammatory status. The plasma visfatin level may influence the risk of CAD (Liu et al., 2009; Kadoglou et al., 2011; Wang et al., 2011a). Therefore, it was hypothesized that plasma visfatin could be functional and might be associated with the development of MI. A number of studies have been conducted to investigate the potential association between the plasma visfatin level and MI risk (Saddi-Rosa et al., 2010; Lu et al., 2012). Recently, several studies have indicated that the plasma visfatin level might play a critical role in increasing MI risk ( $\mathrm{Lu}$ et al., 2012; Mazaherioun et al., 2012). However, some other studies suggest that the plasma visfatin level is not associated with susceptibility to MI. In view of the conflicting results of previous studies, we performed a meta-analysis of all available data to evaluate the association between the plasma visfatin level and susceptibility to MI. 


\section{MATERIAL AND METHODS}

\section{Search strategy}

An extensive literature search was conducted on the PubMed, Embase, Web of Science, Cochrane Library, and CBM databases for relevant articles published from the databases' dates of inception through May 1, 2013. We used the following keywords and MeSH terms: "myocardial infarction", "myocardial infarct", "MI", "AMI", and "visfatin". No language restriction was applied. A manual search of the reference lists from potentially relevant articles was also performed to identify other studies of possible interest.

\section{Selection criteria}

To be included in the meta-analysis, the studies had to meet the following criteria: 1) clinical case-control studies focused on the association between the plasma visfatin level and MI risk; 2) confirmation of the diagnosis of MI by performing clinical examinations, such as electrocardiography, echocardiography, or coronary angiography, in all cases; 3) inclusion of sufficient data regarding the plasma level of visfatin. Studies that did not meet all of the inclusion criteria were excluded. If more than one study by the same author using the same case series had been published, only the study with the largest sample size or the most recent publication was included. Any disagreements were discussed until a consensus was reached.

\section{Data extraction}

Two authors independently extracted data from the eligible studies by using a standardized form. The following information was collected: surname of first author, year of publication, source of publication, country of origin, language of publication, study design, ethnicity of subjects, total number of subjects, gender ratio, mean age, and nature of the sample in which the visfatin level was measured. In cases of conflicting evaluations, disagreements on inconsistent data from the eligible studies were resolved through discussion and careful reexamination of the full text by the authors.

\section{Quality assessment}

The quality of the included studies was assessed independently by two authors based on the Newcastle-Ottawa Scale (NOS) criteria for the assessment of the quality of nonrandomized studies (Jackson et al., 2012). The NOS criteria use a "star" rating system to evaluate the methodological quality based on 3 aspects of the study: selection, comparability, and outcome. Scores range from 0 stars (worst) to 9 stars (best); a score equal to or greater than 7 indicates generally good methodological quality. Disagreements on the assessed quality of the included studies were resolved through comprehensive reassessment by the authors.

\section{Statistical analysis}

Crude standardized mean differences (SMDs) with 95\% confidence intervals (CIs) were 
calculated. The statistical significance of each pooled SMD was examined by using the Z-test. Between-study variations and heterogeneities were estimated by using Cochran's Q-statistic, with a $P$ value $<0.05$ indicating statistically significant heterogeneity (Biggerstaff and Jackson, 2008). We also quantified the effects of heterogeneity by using the $\mathrm{I}^{2}$ test (range $=0$ to $100 \%$ ), which represents the proportion of inter-study variability that can be attributed to heterogeneity rather than to chance (Peters et al., 2006). When a significant Q-test result with $\mathrm{P}<0.05$ or $\mathrm{I}^{2}>50 \%$ indicated the existence of heterogeneity among the studies, a random-effect model (DerSimonian Laird method) was applied for the meta-analysis; otherwise, a fixed-effect model (Mantel-Haenszel method) was used. Sensitivity analysis was performed by omitting each study in turn and assessing the quality and consistency of the results. Begg's funnel plots and the Egger linear regression test were used to evaluate the publication bias (Hu et al., 2008). All reported P values are two-sided. All analyses were performed with the STATA Version 12.0 software (Stata Corp., College Station, TX, USA).

\section{RESULTS}

\section{Baseline characteristics of the studies included}

Application of the inclusion criteria resulted in the inclusion of 11 case-control studies (Hu et al., 2009; Shi et al., 2010; Su et al., 2010; Ji and Cui, 2011; Wang et al., 2011b; Chen et al., 2012; Hao et al., 2012; Jiao et al., 2012; Lu et al., 2012; Mazaherioun et al., 2012; Xu et al., 2012 ) in this meta-analysis and the exclusion of 23 studies. A flow chart of the study selection process is shown in Figure 1. This meta-analysis comprised a total of 684 subjects, including 362 patients with MI and 322 healthy controls. The years of publication of the studies included ranged from 2009 to 2012. The diagnosis of MI was confirmed by performing electrocardiography, echocardiography, or coronary angiography in all cases. The characteristics and levels of methodological quality of the studies included are summarized in Table 1.

\section{Quantitative data synthesis}

Because heterogeneity obviously existed $(\mathrm{P}<0.05)$, a random-effect model was used to pool these results. This meta-analysis showed that an elevated plasma level of visfatin was associated with an increased risk of $\mathrm{MI}(\mathrm{SMD}=3.82,95 \% \mathrm{CI}=2.67-4.98, \mathrm{P}<0.001)$ (Figure 2). Further stratification based on the source of the controls showed that a high plasma visfatin level was significantly associated with increased risk of MI in both hospital-based and population-based studies $(\mathrm{SMD}=4.12,95 \% \mathrm{CI}=2.23-6.01, \mathrm{P}<0.001$ and $\mathrm{SMD}=3.65,95 \% \mathrm{CI}=$ 2.67-4.98, $\mathrm{P}<0.001$, respectively) (Figure 3).

\section{Evaluation of heterogeneity and publication bias}

Sensitivity analysis was performed to assess the influence of each individual study on the pooled estimates by omitting each in turn. The results suggested that no individual study significantly affected the pooled estimates of the associations between the plasma visfatin level and MI risk, indicating that our results were statistically robust (Figure 4). Begg's funnel plots and the Egger linear regression test were applied to assess the publication bias among the studies included. The shapes of the funnel plots of the associations between the plasma level 
of visfatin and MI risk revealed no obvious asymmetry (Figure 5). Furthermore, the Egger test also provided no strong statistical evidence of publication bias $(t=-1.29, \mathrm{P}=0.228)$.

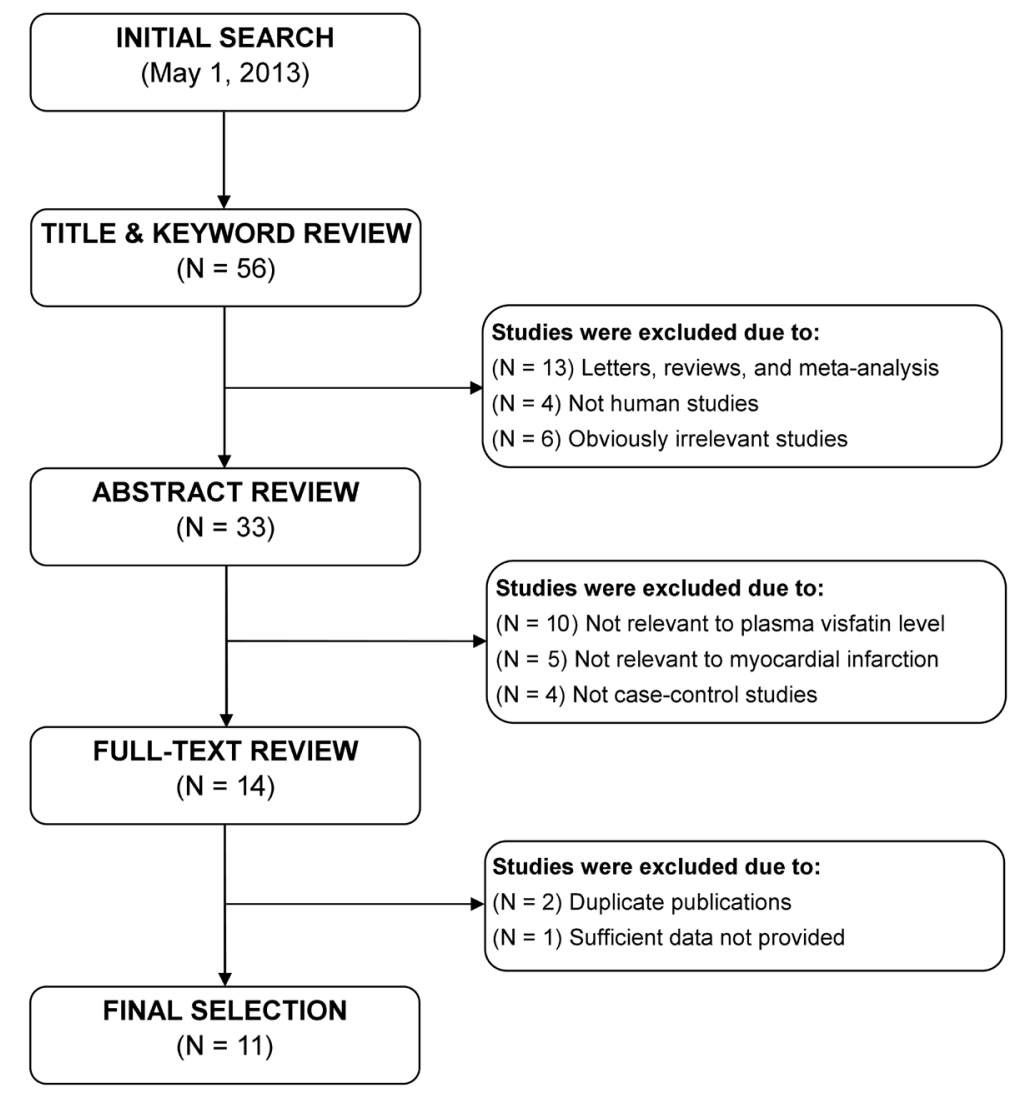

Figure 1. Flow chart of the literature search and study selection process.

Table 1. Main characteristics and levels of methodological quality of all eligible studies.

\begin{tabular}{|c|c|c|c|c|c|c|c|c|c|c|c|c|}
\hline \multirow[t]{2}{*}{ First author } & \multirow[t]{2}{*}{ Year } & \multirow[t]{2}{*}{ Country } & \multicolumn{2}{|c|}{ Number } & \multicolumn{2}{|c|}{ Gender (M/F) } & \multicolumn{2}{|c|}{ Age (years) } & \multicolumn{2}{|c|}{ Source } & \multirow{2}{*}{$\begin{array}{l}\text { Detection } \\
\text { method }\end{array}$} & \multirow{2}{*}{$\begin{array}{l}\text { NOS } \\
\text { scale }\end{array}$} \\
\hline & & & Cases & Controls & Cases & Controls & Cases & Controls & Cases & Controls & & \\
\hline Hu et al. & 2009 & China & 24 & 25 & $15 / 9$ & $14 / 11$ & $66.0 \pm 11.1$ & $62.0 \pm 9.4$ & $\mathrm{HB}$ & $\mathrm{HB}$ & ELISA & 7 \\
\hline Shi et al. & 2010 & China & 27 & 33 & $21 / 6$ & $22 / 11$ & $69.7 \pm 10.3$ & $67.0 \pm 12.7$ & HB & $\mathrm{HB}$ & ELISA & 7 \\
\hline Su et al. & 2010 & China & 20 & 15 & $14 / 6$ & $10 / 5$ & $60.4 \pm 7.3$ & $58.1 \pm 7.4$ & HB & HB & ELISA & 7 \\
\hline $\mathrm{Ji}$ and $\mathrm{Cui}$ & 2011 & China & 30 & 30 & $17 / 13$ & $15 / 15$ & $62.4 \pm 7.5$ & $62.0 \pm 7.1$ & HB & PB & ELISA & 8 \\
\hline Wang et al. & $2011 b$ & China & 20 & 20 & $11 / 9$ & $13 / 7$ & $61.4 \pm 12.3$ & $60.5 \pm 8.4$ & HB & PB & ELISA & 7 \\
\hline Lu et al. & 2012 & China & 54 & 28 & $40 / 14$ & $16 / 12$ & $63.1 \pm 14.1$ & $57.9 \pm 13.3$ & $\mathrm{HB}$ & $\mathrm{HB}$ & EIA & 8 \\
\hline Mazaherioun et al. & 2012 & Iran & 72 & 83 & $56 / 16$ & $59 / 24$ & $61.6 \pm 11.4$ & $60.3 \pm 8.3$ & $\mathrm{HB}$ & PB & ELISA & 8 \\
\hline Chen et al. & 2012 & China & 20 & 20 & - & - & $56.0 \pm 8.7$ & $56.7 \pm 5.3$ & HB & PB & ELISA & 8 \\
\hline Hao et al. & 2012 & China & 33 & 30 & $20 / 13$ & $18 / 12$ & $51.4 \pm 8.0$ & $52.4 \pm 9.6$ & $\mathrm{HB}$ & HB & ELISA & 7 \\
\hline Jiao et al. & 2012 & China & 48 & 20 & $27 / 21$ & $14 / 6$ & $55.0 \pm 8.0$ & $56.0 \pm 6.0$ & HB & PB & ELISA & 8 \\
\hline Xu et al. & 2012 & China & 14 & 18 & $8 / 6$ & $10 / 8$ & $63.5 \pm 10.0$ & $61.3 \pm 8.9$ & $\mathrm{HB}$ & PB & ELISA & 8 \\
\hline
\end{tabular}

$\mathrm{M}=$ male; $\mathrm{F}=$ female; $\mathrm{HB}=$ hospital-based study; $\mathrm{PB}=$ population-based study; ELISA = enzyme-linked immunosorbent assay; EIA = enzyme immunoassay; NOS = Newcastle-Ottawa Scale. 


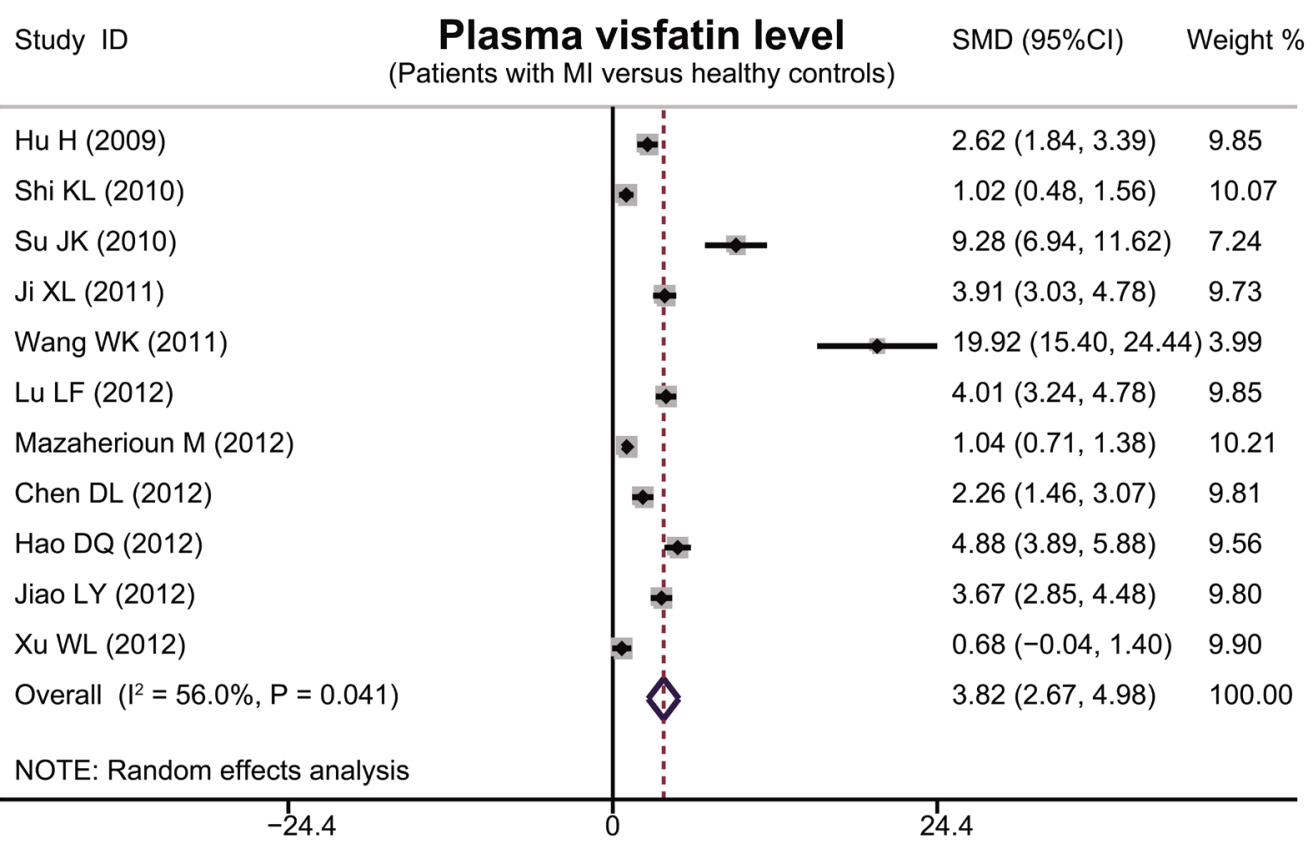

Figure 2. Forest plot of the association between the plasma visfatin level and susceptibility to myocardial infarction. $\mathrm{MI}=$ myocardial infarction; $\mathrm{SMD}=$ standardized mean difference $; 95 \% \mathrm{CI}=95 \%$ confidence interval.

\begin{tabular}{|c|c|c|c|}
\hline \multirow{2}{*}{$\begin{array}{l}\text { Study ID } \\
\text { Hospital-based }\end{array}$} & $\begin{array}{l}\text { Plasma visfatin level } \\
\text { Patients with MI versus healthy controls) }\end{array}$ & \multirow[t]{2}{*}{ SMD $(95 \% \mathrm{Cl})$} & Weight \% \\
\hline & & & \\
\hline Hu H (2009 & & $2.62(1.84,3.39)$ & 9.85 \\
\hline Shi KL (2010) & $\bullet$ & $1.02(0.48,1.56)$ & 10.07 \\
\hline Su JK (2010) & & $9.28(6.94,11.62)$ & 7.24 \\
\hline Lu LF (2012) & & $4.01(3.24,4.78)$ & 9.85 \\
\hline Hao DQ (2012) & & $4.88(3.89,5.88)$ & 9.56 \\
\hline Subtotal $\left(I^{2}=59.0 \%, P=0.036\right)$ & & $4.12(2.23,6.01)$ & 46.57 \\
\hline \multicolumn{4}{|l|}{ Population-based } \\
\hline Ji XL (2011) & + & $3.91(3.03,4.78)$ & 9.73 \\
\hline Wang WK (2011) & & $19.92(15.40,24.44)$ & 4) 3.99 \\
\hline Mazaherioun M (2012) & - & $1.04(0.71,1.38)$ & 10.21 \\
\hline Chen DL (2012) & + & $2.26(1.46,3.07)$ & 9.81 \\
\hline Jiao LY (2012) & + & $3.67(2.85,4.48)$ & 9.80 \\
\hline Xu WL (2012) & + & $0.68(-0.04,1.40)$ & 9.90 \\
\hline Subtotal $\left(I^{2}=53.6 \%, P=0.047\right)$ & & $3.65(2.03,5.28)$ & 53.43 \\
\hline Overall $\left(I^{2}=56.0 \%, P=0.041\right)$ & $\phi$ & $3.82(2.67,4.98)$ & 100.00 \\
\hline NOTE: Random effects analysis & & & \\
\hline-24.4 & 0 & & \\
\hline
\end{tabular}

Figure 3. Subgroup analysis by the source of the controls for the association between the plasma visfatin level and susceptibility to myocardial infarction. $\mathrm{MI}=$ myocardial infarction; $\mathrm{SMD}=$ standardized mean difference; $95 \% \mathrm{CI}$ $=95 \%$ confidence interval. 


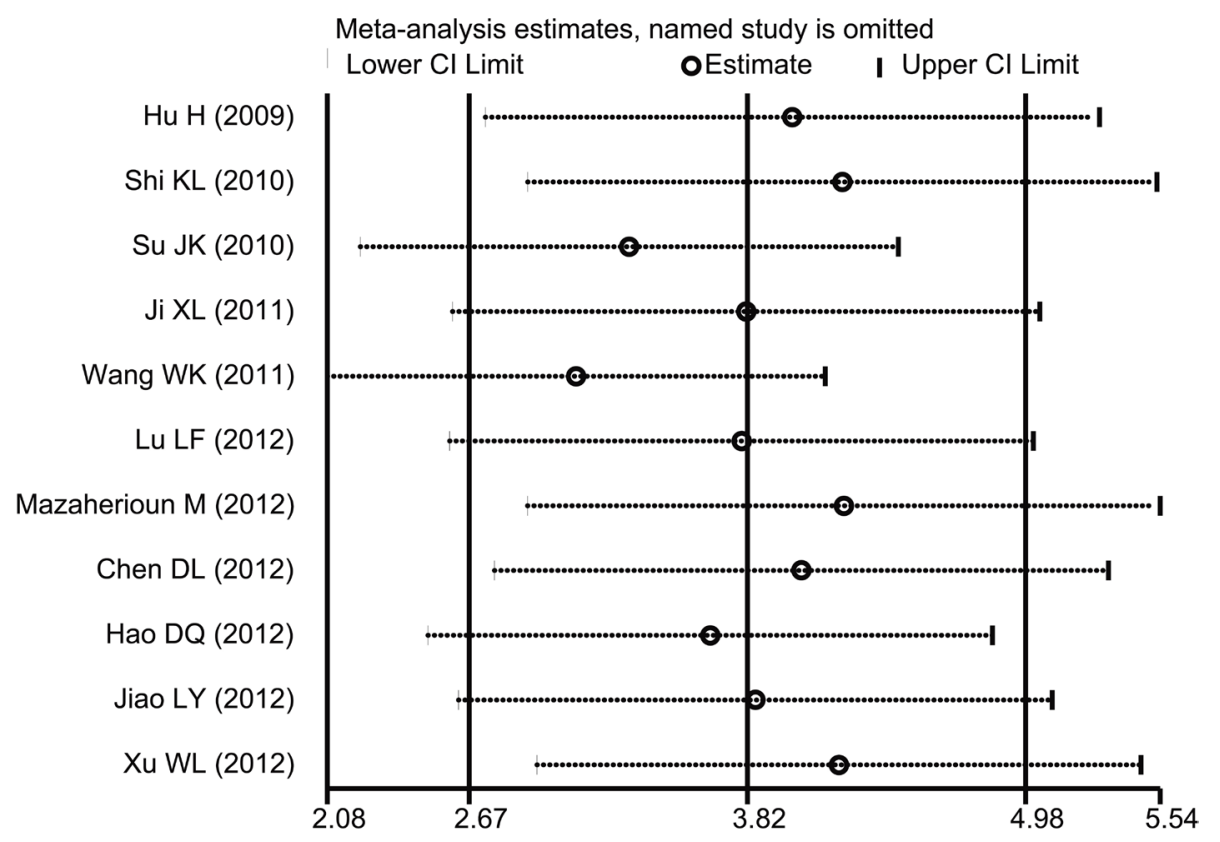

Figure 4. Sensitivity analysis of the summary odds ratio of the association between the plasma visfatin level and susceptibility to myocardial infarction. The results were computed by omitting each study in turn. Meta-analysis random-effect estimates (exponential form) were used. The 2 ends of the dotted lines represent the $95 \% \mathrm{CI}$.
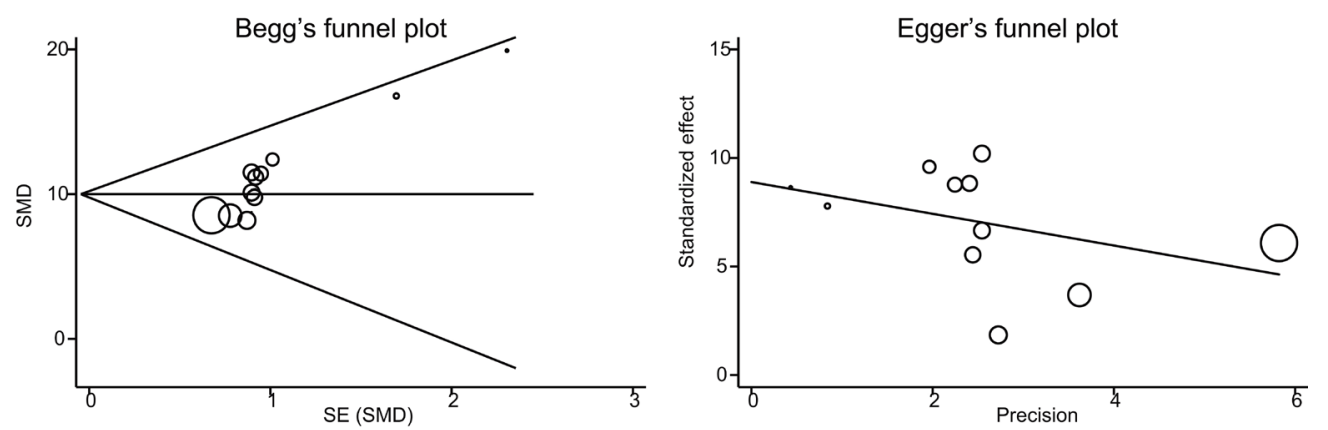

Figure 5. Begg's funnel plot of the associations between the plasma visfatin level and susceptibility to myocardial infarction. Each point represents the indicated association in an individual study. SE = standard error; SMD = standardized mean difference. Horizontal line $=$ mean magnitude of the effect.

\section{DISCUSSION}

Visfatin is a novel adipokine that is secreted predominantly by visceral adipose tissue (Luk et al., 2008). Recently, visfatin has been studied as a multifunctional protein that 
plays important roles in immunity, metabolism, aging, inflammation, and responses to stress (Tilg and Moschen, 2006; Adya et al., 2008). Visfatin participates in several pathophysiological processes that contribute to $\mathrm{CAD}$, including hypertension, atherosclerosis, ischemic heart disease, ischemic stroke, and MI (Wang et al., 2012). Increasingly strong evidence suggests that the plasma visfatin level is associated with the risk of MI and that plasma visfatin may well be a promising biomarker for the diagnosis of MI. The available evidence constitutes a compelling biological rationale for using the plasma level of visfatin for the detection of MI.

In this study, we provide evidence that the plasma visfatin level is significantly higher in patients with MI than in healthy controls. Our results indicate that an elevated plasma level of visfatin may increase the risk of MI, which is consistent with the outcomes of previous studies. Our findings support the existing literature regarding the role of visfatin in the pathogenesis of MI. We strongly believe that visfatin could be used in the future as a reliable biomarker for predicting the development of MI. Although the biological mechanisms by which visfatin participates in the pathogenesis of MI are not well understood, many previous studies have suggested that visfatin could be an important inflammatory protein associated with the destabilization of atherosclerotic plaque that leads to MI. Adya et al. (2008) reported that visfatin is involved in the processes of myocardial recovery and remodeling. Many experimental studies have indicated that visfatin can act via the PI3K-Akt signaling pathway to protect against acute myocardial ischemia-reperfusion injury after acute or chronic hypoxia (Lim et al., 2008; Smith and Yellon, 2011). In the current study, we also performed subgroup analysis to evaluate the roles of visfatin in the development of MI in different populations. The results strongly suggested that measurement of the plasma visfatin level might be a promising technique for the detection and estimation of the prognosis of MI.

Our meta-analysis has several limitations that should be acknowledged. The first major limitation is the relatively small sample size, which may not have provided sufficient statistical power to detect the role of the plasma visfatin level in the development of MI. Therefore, additional studies with larger sample sizes are still needed. On the other hand, a meta-analysis of the summarized data from previously published studies, as a type of retrospective study, may be subject to recall or selection bias that might possibly influence the reliability of the results. Most importantly of all, the lack of access to all of the data from the original studies limited further evaluation of the potential usefulness of the plasma visfatin level. However, despite these statistical limitations, our study is the first comprehensive meta-analysis of all high-quality studies concerning the association between the plasma visfatin level and MI risk.

In conclusion, our meta-analysis suggests that an elevated plasma level of visfatin may contribute to the risk of MI. Plasma visfatin may be a promising biomarker for the detection and estimation of the prognosis of MI. Given the previously mentioned limitations, detailed studies are needed to confirm our findings. Further studies are still warranted to validate the associations between the plasma visfatin level in combination with other risk factors and MI risk.

\section{ACKNOWLEDGMENTS}

Research supported by the Foundation of the Science and Technology Research Project of Liaoning Province (\#2010225036), the Science and Technology Research Project of Shenyang City (\#F10-149-9-47) and the Project of Educational Department of Liaoning Province (\#L2010555). 


\section{REFERENCES}

Adya R, Tan BK, Punn A, Chen J, et al. (2008). Visfatin induces human endothelial VEGF and MMP-2/9 production via MAPK and PI3K/Akt signalling pathways: novel insights into visfatin-induced angiogenesis. Cardiovasc. Res. 78: 356-365.

Akerman MJ, Calacanis CM and Madsen MK (2004). Relationship between asthma severity and obesity. J. Asthma 41: 521-526.

Berg AH and Scherer PE (2005). Adipose tissue, inflammation, and cardiovascular disease. Circ. Res. 96: 939-949.

Biggerstaff BJ and Jackson D (2008). The exact distribution of Cochran's heterogeneity statistic in one-way random effects meta-analysis. Stat. Med. 27: 6093-6110.

Chen DL, Wang LB, Zhao XH and Yue W (2012). Expression of serum hypoxia-inducible factor-1 $\alpha$ and visfatin and their correlation with Gensini score in patients with acute coronary syndrome. Chin. J. Cardiovasc. Rehabil. Med. 21: $255-258$.

Freedman DS, Khan LK, Dietz WH, Srinivasan SR, et al. (2001). Relationship of childhood obesity to coronary heart disease risk factors in adulthood: the Bogalusa Heart Study. Pediatrics 108: 712-718.

Guerre-Millo M (2004). Adipose tissue and adipokines: for better or worse. Diabetes Metab. 30: 13-19.

Hao DQ, Han WJ and Liu HL (2012). Analysis of visfatin and adiponectin in patients with acute coronary syndrome. $J$. Zhengzhou Univ. (Med. Sci.) 47: 373-376.

$\mathrm{Hu} \mathrm{H}$, Zhang HQ, Chen DK and Huang WJ (2009). Clinical value of serum visfatin in patients with coronary artery disease. Chin. J. Arterioscler. 17: 692-694.

$\mathrm{Hu}$ W, Wang Z, Wang H, Huang H, et al. (2008). Serum visfatin levels in late pregnancy and pre-eclampsia. Acta Obstet. Gynecol. Scand. 87: 413-418.

Iacobellis G and Bianco AC (2011). Epicardial adipose tissue: emerging physiological, pathophysiological and clinical features. Trends Endocrinol. Metab. 22: 450-457.

Insam C, Paccaud F and Marques-Vidal P (2013). Trends in hospital discharges, management and in-hospital mortality from acute myocardial infarction in Switzerland between 1998 and 2008. BMC Public Health 13: 270.

Jackson D, White IR and Riley RD (2012). Quantifying the impact of between-study heterogeneity in multivariate metaanalyses. Stat. Med. 31: 3805-3820.

Ji XL and Cui JG (2011). Relationship between plasma visfatin and coronary heart disease and their changes after percutaneous coronary intervention. Mol. Cardiol. China 11: 266-269.

Jiao LY, Wang Y and Yuan Y (2012). The changes and clinical significance of serum visfatin and adiponectin levels in patients with coronary heart disease. Lab. Med. 27: 349-351.

Kadoglou NP, Gkontopoulos A, Kapelouzou A, Fotiadis G, et al. (2011). Serum levels of vaspin and visfatin in patients with coronary artery disease - Kozani study. Clin Chim. Acta 412: 48-52.

Lim SY, Davidson SM, Paramanathan AJ, Smith CC, et al. (2008). The novel adipocytokine visfatin exerts direct cardioprotective effects. J. Cell Mol. Med. 12: 1395-1403.

Liu SW, Qiao SB, Yuan JS and Liu DQ (2009). Association of plasma visfatin levels with inflammation, atherosclerosis and acute coronary syndromes (ACS) in humans. Clin. Endocrinol. 71: 202-207.

Lu LF, Wang CP, Yu TH, Hung WC, et al. (2012). Interpretation of elevated plasma visfatin concentrations in patients with ST-elevation myocardial infarction. Cytokine 57: 74-80.

Luk T, Malam Z and Marshall JC (2008). Pre-B cell colony-enhancing factor (PBEF)/visfatin: a novel mediator of innate immunity. J. Leukoc Biol. 83: 804-816.

Mazaherioun M, Hosseinzadeh-Attar MJ, Janani L, Vasheghani FA, et al. (2012). Elevated serum visfatin levels in patients with acute myocardial infarction. Arch. Iran. Med. 15: 688-692.

Peters JL, Sutton AJ, Jones DR, Abrams KR, et al. (2006). Comparison of two methods to detect publication bias in metaanalysis. JAMA 295: 676-680.

Saddi-Rosa P, Oliveira CS, Giuffrida FM and Reis AF (2010). Visfatin, glucose metabolism and vascular disease: a review of evidence. Diabetol. Metab. Syndr. 2: 21.

Shi KL, Qiu ZH, Zhu Y and Chen Y (2010). The serum visfatin level in coronary artery disease patients and its relationship to coronary artery disease. Chin. J. Clin. Med. 17: 778-780.

Smith CC and Yellon DM (2011). Adipocytokines, cardiovascular pathophysiology and myocardial protection. Pharmacol. Ther. 129: 206-219.

Stephen WC and Janssen I (2009). Sarcopenic-obesity and cardiovascular disease risk in the elderly. J. Nutr. Health Aging 13: 460-466.

Stone GW, Maehara A, Lansky AJ, de Bruyne B, et al. (2011). A prospective natural-history study of coronary 
atherosclerosis. N. Engl. J. Med. 364: 226-235.

Su JK, Dang YH, He F and Li L (2010). Detection of plasma visfatin level in patients with coronary heart disease. Clinical Focus 25: 185-187.

Tilg H and Moschen AR (2006). Adipocytokines: mediators linking adipose tissue, inflammation and immunity. Nat. Rev Immunol. 6: 772-783.

Wang LS, Yan JJ, Tang NP, Zhu J, et al. (2011a). A polymorphism in the visfatin gene promoter is related to decreased plasma levels of inflammatory markers in patients with coronary artery disease. Mol. Biol. Rep. 38: 819-825.

Wang P, Xu TY, Guan YF, Su DF, et al. (2009). Perivascular adipose tissue-derived visfatin is a vascular smooth muscle cell growth factor: role of nicotinamide mononucleotide. Cardiovasc. Res. 81: 370-380.

Wang P, Vanhoutte PM and Miao CY (2012). Visfatin and cardio-cerebro-vascular disease. J. Cardiovasc. Pharmacol. 59: 1-9.

Wang WK, Mou CP, Yang GY and Mou XL (2011b). Association between visfatin and thrombomodulin proteins with acute coronary syndrome. Helongjiang Med. Pharm. 34: 63-64.

$\mathrm{Xu}$ WL, Li JH, Zhang CQ, Shao YB, et al. (2012). Serum visfatin and its influencing factors in patients with coronary artery disease. Chin. Circul. J. 27: 137-140.

Yan JJ, Tang NP, Tang JJ, Jia EZ, et al. (2010). Genetic variant in visfatin gene promoter is associated with decreased risk of coronary artery disease in a Chinese population. Clin. Chim. Acta 411: 26-30.

Yeh RW, Sidney S, Chandra M, Sorel M, et al. (2010). Population trends in the incidence and outcomes of acute myocardial infarction. N. Engl. J. Med. 362: 2155-2165.

Zhang H, Cui J and Zhang C (2010). Emerging role of adipokines as mediators in atherosclerosis. World J. Cardiol. 2: 370-376. 